Meta

Journal des tradlucteurs

Translators' Journal

\title{
Commuter : un mot pour tout dire
}

\section{Charles Dupont}

Volume 30, numéro 4, décembre 1985

URI : https://id.erudit.org/iderudit/002679ar

DOI : https://doi.org/10.7202/002679ar

Aller au sommaire du numéro

Éditeur(s)

Les Presses de l'Université de Montréal

ISSN

0026-0452 (imprimé)

1492-1421 (numérique)

Découvrir la revue

Citer cet article

Dupont, C. (1985). Commuter : un mot pour tout dire. Meta, 30(4), 379-384.

https://doi.org/10.7202/002679ar d'utilisation que vous pouvez consulter en ligne.

https://apropos.erudit.org/fr/usagers/politique-dutilisation/ 


\section{COMMUTER : UN MOT POUR TOUT DIRE}

Le terme commuter est fréquemment employé dans les textes concernant l'aviation civile et ce, dans des sens non encore consignés dans les dictionnaires généraux de langue anglaise. Ce mot étatsunien a presque totalement remplacé l'expression third level carrier, traduite en France à la fin des années 60 par transporteur de troisième niveau "parce qu'on n'avait pas trouvé mieux alors que de traduire l'expression américaine pour désigner le transport aérien régional naissant $» 1$.

Curieusement les désignations avec level, si on fait exception de third level qui figure dans certains dictionnaires anglais, sont disparues de l'usage aux États-Unis, alors qu'en français l'expression troisième niveau est toujours courante et que celle de deuxième niveau est parfois employée pour désigner un grand transporteur intérieur.

La traduction de commuter pose souvent des difficultés pour lesquelles le Harrap's et le Robert-Collins, encore restés à la notion de banlieusard, sont d'aucuns secours. Le présent article vise à faire la lumière sur les divers usages du vocable commuter et à établir les divers équivalents possibles suivant les contextes.

Le terme commuter, comme substantif ou adjectif, peut en effet désigner un transporteur aérien, un avion et même un passager, ou encore qualifier un vol, un service et une industrie. Ce terme polysémique en aviation commerciale est difficile à bien cerner et à traduire par suite du fait qu'il ne s'agit pas d'une catégorie officielle de transporteurs ou d'un type très précis d'avions. Par conséquent, la notion à laquelle se réfêre chaque auteur est devenue passablement floue. Pour illustrer cette confusion, citons des définitions tirées d'un dictionnaire anglais ${ }^{2}$ récent :

commuter - airline which does not operate large aircraft and performs between two or more points and publishes flight schedules.

commuter air carrier - (1) an airliner operating a small aircraft on an irregular basis to serve small communities. (2) Air carriers with the role of connecting service to hub airports (see also commuter airline).

commuter air carrier operator - (1) an air taxi operator which performs round trips per week between two or more points and publishes fight schedules which specify the times, days of the week and places between which such flights are operated. (2) An air taxi operator which has a contract with the U.S. Postal Service to transport mail by air.

commuter airline - an air taxi which operates with passengers, cargo, or mail on a prescribed route and schedule.

commuter operator - operators of light aircraft who perform scheduled round trips between two or more points or carry mail.

Tout cela ne peut que nous laisser perplexe sur le sens de commuter, notamment parce que le seul sens retenu est celui de transporteur. Le dictionnaire cité est également muet sur les termes feeder, connector et third level carrier souvent employés comme synonymes ou quasi synonymes de commuter. Pour l'instant, il vaut mieux s'en tenir à une 
définition plus générale tirée du Jane's Aerospace Dictionary :

commuter airline - in theory, air carrier operating between outlying regions and major $h u b(s)$. In practice, applied to anything from air-taxi operator to - in undeveloped regions - national carrier ${ }^{3}$

Un peu d'histoire ... étatsunienne. Au début des années 60 , les petits transporteurs étaient connus aux États-Unis sous les appellations de local airlines et de scheduled air taxis lesquels étaient aussi appelés third level carriers et feeders. Insatisfaits de leurs appellations, ces derniers devinrent peu après des commuter airlines. En 1974, ils formèrent la Commuter Airline Association of America (CAAA), laquelle fut renommée Regional Airline Association (RAA) en 1982, non sans opposition comme le prouve le commentaire suivant :

After NTSB and FAA called commuters " unsafe and unacceptable risk", in 1978 many of the larger and influential carriers felt the name "commuter" was tainted. They felt this could be corrected by simply changing the CAAA to the Regional Airline Association. /.../ May smaller operators objected to the plan because they felt the name commuter was more ideally descriptive of the special role of these short-haul carriers in the U.S. ${ }^{4}$

Il faut aussi rappeler que l'adoption du Airline Deregulation Act en 1978 aux États-Unis a complètement bouleversé les appellations des différentes catégories de transporteurs homologués, catégories fondées sur les recettes d'exploitation. Ainsi, les catégories trunk, local, regional, commuter et supplemental sont disparues au profit de major, national, large regional et medium regional (à noter que même si small regional n'existe pas, cela n'empêche pas la revue Air Transport World de publier ses statistiques " commuter" en divisant les transporteurs en large regionals et small regionals, distinction fondée cette fois sur le nombre de passagers transportés.

L'évolution sémantique continue toutefois aux États-Unis, si l'on en juge par la déclaration de Stephen Smith de la RAA dans Aéroports magazine en 1982 :

Nous pensons, qu'à l'avenir, la classification des compagnies américaines s'opérera en fonction des routes qu'elles desservent : long-courriers, moyen-courriers, court-courriers /.../ les commuters sont des compagnies court-courriers. Quant à définir les limites du courtcourrier, nous l'étudions...5

Le fait qu'un article en décembre 1984 dans Air Transport World était intitulé "1964-1984 : Commuter/regionals blossomed as short-haul carriers "6 semble confirmer cette évolution.

On peut, grâce aux traits notionnels et aux éléments d'histoire vus jusqu'ici, résumer en disant que le terme commuter est devenu un mot commode pour dénommer les transporteurs exploitant de "petits" avions sur de courtes liaisons intérieures, mais dont le rôle ou la nature des services peuvent varier suivant les créneaux exploités ou l'organisation des transports aériens dans un pays. Voyons maintenant les divers sens et usages de commuter et les équivalents possibles en français.

On trouve d'abord le terme commuter dans les sens de transporteur. Les caractéristiques les plus souvent mentionnées pour les commuters sont :

1. le type d'appareils exploités (sens étudié plus loin) ;

2. la nature des liaisons aériennes (entre deux petites localités ou entre une localité et un grand aéroport d'un même pays);

3. la nature régulière du service ;

4. la distance des liaisons aériennes offertes (longueur toujours inférieure à 1000 $\mathrm{km}$ avec une forte proportion pour des distances de 200 à $500 \mathrm{~km}$ ); 
5. un nombre minimal de vols (norme peu uniforme).

Outre ces caractéristiques de base, on reconnaît généralement que le service des commuters comporte deux dimensions : l'une relative à des services d'apport et l'autre relative au transport régional. Cela explique en partie pourquoi ces transporteurs sont souvent désignés par l'expression commuter/regional carrier.

$\mathrm{Si}$, à l'origine, les commuters avant la lettre avaient une vocation régionale, celleci s'est rapidement transformée, du moins aux États-Unis, en une vocation d'apport, c'est-à-dire l'acheminement de passagers vers de grands aéroports pour un vol de correspondance moyen- ou long-courrier ( $70 \%$ des passagers des commuters aux ÉtatsUnis en 1979). Cette notion d'apport donne lieu à une particularité tant en anglais qu'en français.

Pour des raisons évidentes, les grands transporteurs internationaux et intérieurs cherchent à établir des ententes de complémentarité avec les compagnies d'apport qui vont permettre des avantages commerciaux mutuels. D'une part les grands transporteurs drainent une clientèle qu'ils ne peuvent rejoindre directement dans les petites localités et, d'autre part, les compagnies d'apport retirent des avantages en assurant pour les grands transporteurs un service d'éclatement à partir des grands aéroports. C'est la notion du feeding.

On parlera alors en anglais de feeder carrier et en français de transporteur aérien complémentaire, termes quasi synonymes de commuter et transporteur d'apport respectivement. Cette orientation vers les ententes de complémentarité semble actuellement un mouvement irréversible dans beaucoup de pays et presque une question de survie pour les commuters étatsuniens.

Pour ce qui concerne la dimension de transport régional les équivalents sont bien établis : regional air carrier et transporteur aérien régional. Mais comme nous l'indiquons dans le tableau à la fin, le terme français peut aussi traduire commuter si on tient compte de la remarque suivante :

Now that regionals are joining up with the major carriers, use of the name commuter is coming-back?

Nous avons mentionné plus haut que l'expression transporteur de troisième niveau demeurait courante en français sans qu'on puisse y trouver une raison autre qu'une vieille habitude. En effet, ces niveaux n'existent pas dans la réglementation et il est peu logique de parler d'un troisième niveau quand on ne peut identifier les premier et deuxième.

Enfin, si, comme nous le mentionnions dans la partie précédente, l'expression short-haul carrier devait supplanter commuter dans l'usage, le français aurait recours à transporteur court-courrier que nous connaissons déjà. Nous doutons toutefois de cette possibilité. Le terme short tout comme l'expression third level ne sont pas très bons pour les besoins de l'image commerciale ; commuter fait tellement plus dynamique ! En matière de terminologie il ne faut jamais minimiser l'élément connotatif des vocables.

Commuter s'emploie aussi dans le sens d'avion. On a dit plus tôt que les transporteurs " commuters " exploitaient des appareils dont les caractéristiques leur étaient imposées par voie de réglementation. Les critères les plus souvent mentionnés pour définir l'avion " commuter» sont :

a) la masse au décollage (établie par la Federal Aviation Administration (FAA) à $12500 \mathrm{lb} / 5700 \mathrm{~kg}$, mais annulée par le Airline Deregulation Act);

b) le nombre maximal de places (aux Êtats-Unis ce nombre est passé de 30 à 60 lors de l'adoption du Airline Deregulation Act et remplaçait le critère de la masse au décollage); 
c) l'altitude à laquelle l'avion est autorisé à voler.

Ces critères varient d'un pays à l'autre, sont quelque peu arbitraires et changent constamment pour des raisons technologiques, politiques ou autres. Par exemple, en France dans les années 70, la capacité maximale des avions exploités par les transporteurs aériens régionaux avait été fixée à 50 places tout simplement parce que le plus petit appareil d'Air Inter (transporteur intérieur ou de deuxième niveau) avait 50 places.

On s'entend aujourd'hui pour dire que les appareils " commuters» offrent entre 5 et 80 places; il y a quelques années, la majorité se situaient dans la gamme des 10 à 30 places, mais maintenant la capacité tend à évoluer vers l'avion de 50 places. Au-delà de 80 et jusqu'à 180 places, on parlera plutôt d'un avion moyen-courrier, capacité maximale souhaitable pour le moyen-courrier du futur selon l'Association of European Airli$n e s^{8}$. Les avions de capacité supérieure appartiennent à la catégorie des long-courriers.

Les expressions françaises les plus courantes pour traduire commuter sont avion de transport régional, avion court-courrier, petit-porteur et avion d'apport (voir le tableau pour d'autres variantes).

A notre avis, l'expression avion de transport régional a le désavantage d'être longue ; on pourrait par contre utiliser le sigle ATR (tout comme on a abrégé train à grande vitesse en TGV qui est devenu un mot en soi). ATR est cependant identifié à une marque de commerce avec l'avion ATR 42 fabriqué par Aérospatiale et Aeritalia; cela pourrait, pour des raisons non linguistiques, bloquer son emploi, mais il peut arriver qu'un terme créé comme identification commerciale n'ait pu faire l'objet d'une protection et passe dans la langue (le cas de jardinerie est un exemple récent). On a pu aussi noter l'emploi de l'expression avion régional , mais cela nous semble un raccourci peu souhaitable.

Le terme avion court-courrier est employé de plus en plus fréquemment et se justifie par les qualités techniques des nouveaux appareils exploités par les transporteurs aériens régionaux. Son succès dépendra sans doute de celui de son équivalent short-haul. Le parallélisme linguistique est une autre force qui influe sur l'usage.

Petit-porteur, par analogie à gros-porteur qui est passé dans l'usage et à moyenporteur qu'on voit occasionnellement, est une autre expression bien formée mais comme la clientèle est parfois réticente à passer d'un gros avion à un petit, cette expression demeurera toujours marginale.

L'expression avion d'apport est également bien formée, mais elle demeure obscure et sans attrait sur les plans communicationnel et commercial.

Comme on l'a dit au début, le mot commuter s'emploie également dans d'autres sens ou expressions pour décrire d'autres aspects de l'aviation.

Il peut désigner par exemple un passager :

The air commuter is in fact an air traveller who may be visiting an urban centre or another community for a day or two for various reasons or continuing on the main leg of a continental or international journey?.

Il s'agit en fait de passagers intérieurs, par opposition aux passagers internationaux ; cette expression pourrait cependant ne pas être suffisamment précise puisque les passagers des grands transporteurs intérieurs (en principe le deuxième niveau) sont aussi de cette catégorie. Si le contexte l'exige il faut donc employer des syntagmes comme passager des lignes régionales ou des lignes d'apport ou des lignes complémentaires, selon la nature du service décrit ou le degré de précision qu'exige le texte (voir le tableau pour d'autres expressions également possibles).

Les deux dernières expressions qu'on peut signaler sont commuter flight et commuter industry. Il va sans dire que les équivalents français, qui figurent dans le tableau à 
la fin, tiennent compte des éléments signalés plus tôt. Une dernière remarque serait à faire sur l'expression transport aérien interrégional relevée dans une revue ${ }^{10}$ comme synonyme de transport aérien régional. Cette expression peut se justifier du point de vue géographique, mais elle risque de créer de la confusion avec les services des transporteurs intérieurs qui relient justement les régions d'un pays.

TABLEAU RÉCAPITULATIF DES ÉQUIVALENTS POSSIBLES DU TERME COMMUTER

1.

\section{Sens de transporteur (suivant le contexte)}

$$
\text { commuter }
$$

1) transporteur ${ }^{(a)}$ aérien ${ }^{(b)}$ complémentaire (E.-U.)

2) transporteur aérien d'apport (ailleurs)

3) transporteur aérien régional transporteur aérien court-courrier (génériques) syn.

quasi-syn.

syn.

3.

4.

commuter air carrier commuter airline

large and medium regionals $(\mathrm{c})$
feeder
transporteur aérien complémentaire
feeder airline
regional air carrier
transporteur aérien régional
third level carrier
transporteur de troisième niveau

Sens d'avion (équivalents classés suivant leur fréquence notée)

commuter

commuterliner

feederliner

commuter aircraft avion de transport régional (ATR)

(avion) court-courrier

petit-porteur

avion (de lignes) d'apport

avion de troisième niveau

mini-avion de ligne

Sens de passager (suivant le découpage en 1)

air commuter

1) passager des lignes complémentaires passager des services aériens complémentaires

2) passager des lignes d'apport passager des services aériens d'apport

3) passager des lignes régionales passager des services aériens régionaux

Sens d'industrie (suivant le découpage en 1)

commuter industry

1) transport aérien d'apport

2) transport aérien complémentaire

3) transport aérien régional 
5. Sens de vol (suivant le découpage en 1) commuter flight vol complémentaire vol d'apport vol régional vol court-courrier

(a) Le terme transporteur peut être remplacé par compagnie dans chaque cas.

(b) Le terme "aérien" peut être omis dans la majorité des expressions.

(c) Catégorie établie par le Airline Deregulation Act aux États-Unis.

\section{CHARLES DUPONT}

\section{RÉFÉRENCES}

1. SOBOL, Claudine (1975) : « Où en est le troisième niveau ", in Science et Vie, no 690, p. 86.

2. OCRAN, E.B. (1984) : Dictionary of Air Transport and Traffic Control, London, Granada, p. 55.

3. GUNSTON, Bill (1980) : Jane's Aerospace Dictionary, London, Jane's, p. 92.

4. DAVIS, Lou (1984) : «1964-1984 : Commuter/Regionals Blossomed as Short-Haul Carriers", in Air Transport World, décembre, p. 71.

5. SCHRODT, Anita (1982) : "États-Unis : un essor lié à la déréglementation », in Aéroports magazine, $\mathrm{n}^{\circ}$ 127, p. 3 .

6. DAVIS, Lou (1984) : idem, p. 70.

7. DAVIS, Lou (1984) : idem, p. 71.

8. «Moyens courriers futurs : les compagnies aussi ont des idées ", in Air et Cosmos, $\mathrm{n}^{0} 1054,22$ juillet 1985, p. 29.

9. "Commuter Airlines in the 1980. How Big? How Small ? What is a 'Commuter' ?", in Interavia, vol. XXXVI, février 1981, p. 117.

10. "La bonne santé du transport aérien inter-régional : à quel prix ? ", in Air et Cosmos, no 1034, 2 février 1985 , p. 56.

\section{AUTRES RÉFÉRENCES}

DACHARRY, Monique (1981) : Géographie du transport aérien, Paris, Librairies techniques (Litec). BLOCH, André (1981) : "Dossier 'commuters' ", in Aviation 2000, no 70, pp. 9-15.

WALTER, Claire (1981) : "Airline Classifications - The Names aren't the Same Anymore ", in Airport Services Management, p. 50. 